University of Nebraska - Lincoln

DigitalCommons@University of Nebraska - Lincoln

\title{
Energy and Angular Distribution of Electrons Ejected from Hydrogen and Helium Gas by Protons
}

\author{
M. Eugene Rudd \\ University of Nebraska - Lincoln, erudd@unl.edu \\ Theodore Jorgensen, Jr. \\ University of Nebraska - Lincoln
}

Follow this and additional works at: https://digitalcommons.unl.edu/physicsrudd

Part of the Physics Commons

Rudd, M. Eugene and Jorgensen, Jr., Theodore, "Energy and Angular Distribution of Electrons Ejected from Hydrogen and Helium Gas by Protons" (1963). M. Eugene Rudd Publications. 17.

https://digitalcommons.unl.edu/physicsrudd/17

This Article is brought to you for free and open access by the Research Papers in Physics and Astronomy at DigitalCommons@University of Nebraska - Lincoln. It has been accepted for inclusion in M. Eugene Rudd Publications by an authorized administrator of DigitalCommons@University of Nebraska - Lincoln. 


\title{
Energy and Angular Distribution of Electrons Ejected from Hydrogen and Helium Gas by Protons*†
}

\author{
M. Eugene Rudd $\ddagger$ and Theodore Jorgensen, JR. \\ University of Nebraska, Lincoln, Nebraska
}

(Received 18 March 1963)

\begin{abstract}
Differential cross sections for ejection of secondary electrons of various energies at various angles were measured for hydrogen gas bombarded by $100-\mathrm{keV}$ protons and for helium gas bombarded by 50 -, 100-, and $150-\mathrm{keV}$ protons. The range of angles investigated was $10^{\circ}$ to $160^{\circ}$ and the range of electron energies was 1 to $500 \mathrm{eV}$. A unique fixed-port, double-walled scattering chamber was used. Electrons were counted by an electron multiplier after passing through a $127^{\circ}$ electrostatic analyzer. The efficiency of the detector was determined by replacing the analyzer and multiplier by a Faraday cup and making absolute measurements of cross sections differential only in angle. Comparison with the integral of the differential cross sections over all electron energies gave a value of about $78 \%$ for the efficiency. As a function of electron energy the cross sections decrease monotonically above about $2.5 \mathrm{eV}$ and are uncertain below this value. All cross sections decrease monotonically with an increase in angle but are relatively constant above about $110^{\circ}$. The differential cross sections have been integrated in various ways to obtain distributions over electron energy and angle, total cross sections for ionization, average energies of the ejected electrons, and the stopping cross sections due to ionization. Comparisons are made with other experimental results and with theoretical treatments by the Born approximation and the Gryzinski classical theory.
\end{abstract}

\section{INTRODUCTION}

$T^{1}$ $\mathrm{HE}$ ionization of atomic systems by fast protons has been investigated theoretically ${ }^{1-4}$ and experimentally. ${ }^{5-14}$ Most of the experimental work has been directed towards measurement of total-ionization cross sections. Blauth ${ }^{13}$ has measured the energy distribution of the ejected electrons and Kuyatt and Jorgensen ${ }^{14}$ (hereafter referred to as $\mathrm{KJ}$ ) have made the only complete measurements of the angular and energy dependence of the differential cross sections. These measure-

* Supported in part by the U. S. Atomic Energy Commission.

$\dagger$ This report is based in part on a thesis submitted by M.E.R. to the University of Nebraska in partial fulfillment of the requirements for the Ph.D. degree.

$\ddagger$ Present address: Department of Physics, Concordia College, Moorhead, Minnesota.

${ }^{1}$ D. R. Bates and G. Griffing, Proc. Phys. Soc. (London) A66, 961 (1953).

${ }^{2}$ R. A. Mapleton, Phys. Rev. 109, 1166 (1958).

${ }^{3}$ D. R. Bates, M. R. C. McDowell, and A. Omholt, J. Atmos pheric Terrest. Phys. 10, 51 (1957).

${ }^{4}$ M. R. C. McDowell and G. Peach, Phys. Rev. 121, 1383 (1961).

${ }^{5}$ J. P. Keene, Phil. Mag. 40, 369 (1949)

${ }^{6}$ Ia. M. Fogel', L. I. Krupnik, and B. G. Safronov, Zh. Eksperim. i Teor. Fiz. 28, 589 (1955) [translation: Soviet Phys.-JETP 1, 415 (1955)].

${ }^{7}$ H. B. Gilbody and J. B. Hasted, Proc. Roy. Soc. (London) A240, 382 (1957).

${ }^{8}$ V. V. Afrosimov, R. N. Il'in, and N. V. Fedorenko, Zh. Eksperim. i Teor. Fiz. 34, 1398 (1958) [translation: Soviet Phys.JETP 7, 968 (1958)]

${ }^{\circ}$ F. Schwirzke, Z. Phyzik 157, 510 (1960)

${ }^{10}$ N. V. Fedorenko, V. V. Afrosimov, R. N. Il'in, and E. S. Solov'ev, in Proceedings of the Fourth International Conference on Ionization Phenomena in Gases, Uppsala, 1959 (North-Holland

Publishing Company, Amsterdam, 1960), p. IA 47.

${ }^{11}$ J. W. Hooper, E. W. McDaniel, D. W. Martin, and D. S. Harmer, Phys. Rev. 121, 1123 (1961).

${ }_{12}$ E. W. McDaniel, J. W. Hooper, D. W. Martin, and D. S. Harmer, in Proceedings of the Fifth International Conference on Ionization Phenomena in Gases, Munich, 1961 (North-Holland

Publishing Company, Amsterdam, 1962), Vol. I, p. 60.

${ }^{13}$ E. Blauth, Z. Physik 147, 228 (1957).

${ }_{14}$ C. E. Kuyatt and T. Jorgensen, Jr., Phys. Rev. 130, 1444 (1963). ments were made with hydrogen gas bombarded by protons of 50- to $100-\mathrm{keV}$ energy. The present investigation was undertaken to extend these measurements to helium gas. Measurements were also made with hydrogen gas to compare with those of $\mathrm{KJ}$.

In the present work, the range of angles investigated was $10^{\circ}$ to $160^{\circ}$ and the range of energies of the electrons observed was $1 \mathrm{eV}$ to about $500 \mathrm{eV}$. Helium was bombarded with 50-, 100-, and $150-\mathrm{keV}$ protons while measurements were made on hydrogen only at $100 \mathrm{keV}$. Absolute values of ionization cross sections differential in both energy and angle were measured; and by numerical integration, cross sections differential in electron energy only and in angle only have been obtained. By a second integration, total ionization cross sections for each proton energy were also obtained.

\section{EXPERIMENTAL METHOD}

The arrangement of apparatus for the experiment was quite similar to that of $\mathrm{KJ}$ and some of the same equipment was used. However, a new scattering chamber, Fig. 1, was built. The chamber consists of two 4-in.-high concentric brass cylinders of 5- and $9 \frac{5}{8}$-in. o.d. forming an inner and an outer chamber. A vacuum is maintained in the outer chamber while the gas to be bombarded is admitted to the inner chamber. A proton port allows the proton beam from the Nebraska Cockcroft-Walton accelerator to enter the inner chamber. One of two Faraday cups collects the beam. Both cups, biased positively at $67 \frac{1}{2} \mathrm{~V}$, are surrounded by grounded shields to prevent electric fields from appearing in the scattering region. The larger of the two cups was used for all measurements, except those at $10^{\circ}$ when it was retracted and the smaller cup used. Tests indicated that either cup collected the entire proton beam.

An electron pipe is inserted through the outer chamber into the inner chamber to allow the secondary 
Fig. 1. Top view of scattering chamber, analyzer, and detector.

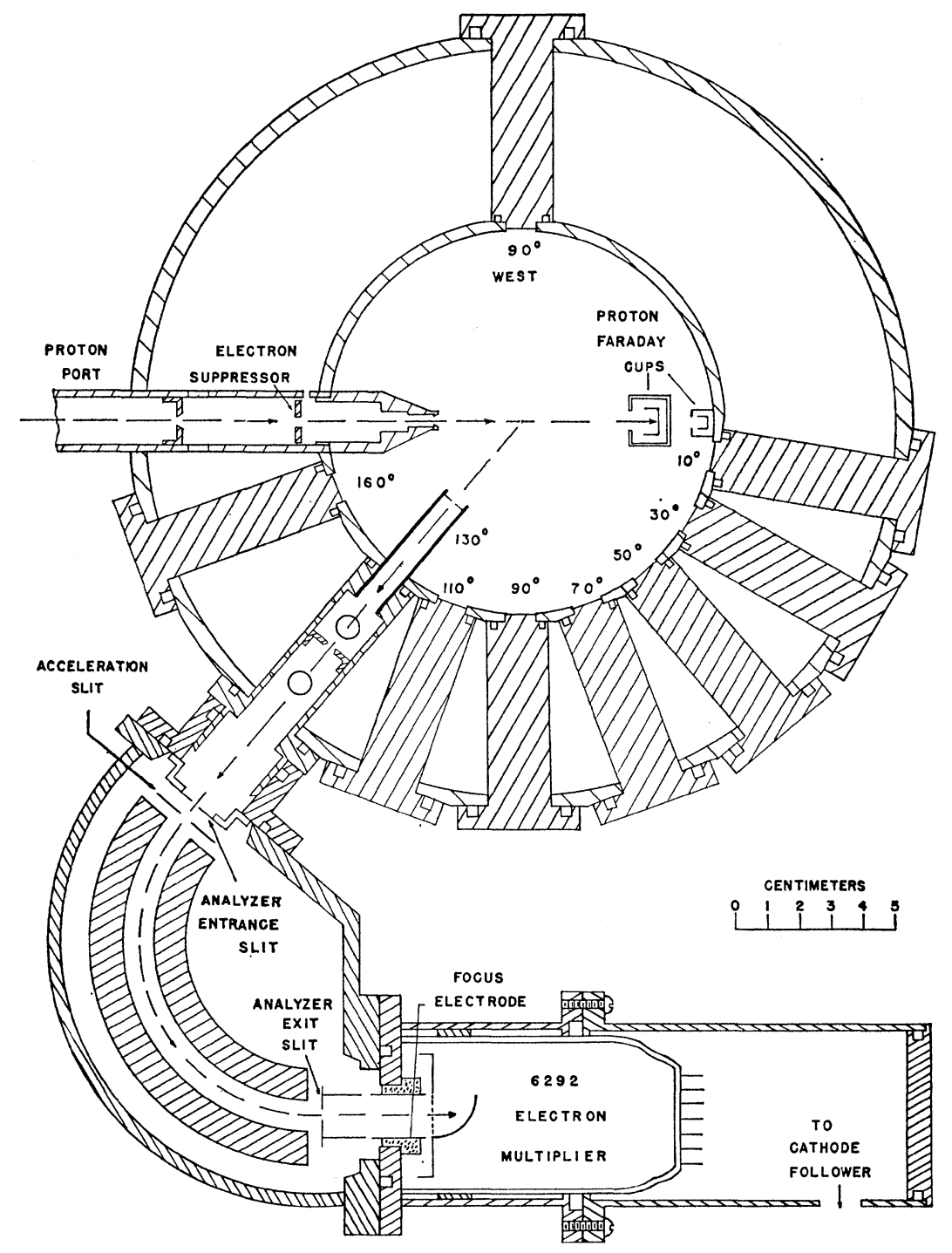

electrons produced in the gas to go to the analyzer and the detector where individual electrons are counted. The electron pipe may be placed in any one of the nine electron ports. Eight of these are placed on one side of the chamber at the $10^{\circ}, 30^{\circ}, 50^{\circ}, 70^{\circ}, 90^{\circ}, 110^{\circ}, 130^{\circ}$, and $160^{\circ}$ positions. An additional port at $90^{\circ}$ on the opposite side of the chamber was provided to check for symmetry and also to allow the insertion of an electron gun for testing the analyzer and detector. The electron ports were machined at angles accurate to $1 / 30^{\circ}$. The ports not occupied by the electron pipe were sealed by brass plugs. The spacing between the O-ring surfaces on the inner and outer chambers was maintained during machining to within 0.001 in. so that proper sealing could be effected without difficulty when the electron pipe was moved from one port to the other. The spacing between these O-ring surfaces and the axis of the chamber was maintained to the same accuracy and the proton port was machined at the same time at the proper angle. Thus, alignment problems were virtually eliminated at the time of construction.

One obvious disadvantage of this type of scattering chamber is that the vacuum must be lost to change angles. In the present work this was not a serious drawback since several runs had to be made at each angle using the two different gases and different proton energies. When the analyzer and detector were to be moved to a different port, the pumping system was valved off and helium gas was admitted to the chamber, analyzer, and detector at a pressure slightly greater than atmospheric. This was done to prevent air from reaching the electron multiplier dynodes. After a roughing pump had reduced the chamber pressure to about $20 \mu$, the valve to the diffusion pump was reopened and generally within $2 \mathrm{~h}$ the pressure was low enough to resume measurements at the next angle. 
The magnetically analyzed proton beam from the accelerator is collimated by two circular apertures, 0.357 and $0.159 \mathrm{~cm}$ in diameter, the second of which is shown in Fig. 1. These apertures have sharp edges to minimize the scattering of the proton beam and the addition of secondary electrons to the beam. To prevent any secondary electrons from entering the chamber with the beam, a suppressor diaphragm biased at $-67 \frac{1}{2} \mathrm{~V}$ was placed in the proton pipe after the second defining aperture.

The current to the proton cup was integrated by a $1.00 \mu \mathrm{F}$ polystyrene capacitor connected between the input and output of a high-gain operational amplifier. The accumulated charge was then read on a $\frac{1}{4} \%$ lowimpedance voltmeter. The beam-current integrator and the scaler which counts the electrons are switched on and off simultaneously. Either $\frac{1}{2}$ or $1 \mu \mathrm{C}$ of charge was collected during each measurement and the time of collection varied from about 12 to $50 \mathrm{sec}$. The beam current ranged from $10^{-8}$ to $8 \times 10^{-8} \mathrm{~A}$.

The $127^{\circ}$ electrostatic analyzer used was the same one described by $\mathrm{KJ}$ except for somewhat larger slits. The constant of the analyzer (the ratio of the voltage across the analyzer plates to the electron energy passed by the analyzer) was remeasured with the new slits and found to be within $\frac{1}{2} \%$ of the calculated value of 0.3646 . The shape of the resolution curve for the analyzer was measured for various electron energies from 6 to $250 \mathrm{eV}$ and in most cases was found to be very close to the calculated trapezoidal shape ${ }^{15}$ with a base width of $0.101 E$ and a top width of $0.013 E$, where $E$ is the electron energy.

During the cross-section measurements the electrons were accelerated just prior to entering the electrostatic analyzer as in the work of KJ and others. This was done because small electric and magnetic fields deflect slowmoving electrons sufficiently to cause a sizeable decrease in counting rate and a noticeable change in the constant of the analyzer. To find the best accelerating voltage a set of curves was run in which the counting rate was plotted against the analyzer voltage while varying the accelerating voltage to pass electrons of a given energy. This yielded curves very similar to those of Fig. 2 of KJ. Because slits of different sizes were used, the best accelerating voltage appeared to be $15 \mathrm{~V}$ instead of the $10 \mathrm{~V}$ used by KJ. The curves also indicated that below about 3-eV electron energy some distortion is caused by the acceleration, probably due to the focusing effect. To insure that no distortion occurred at high energies, cross sections were measured for various accelerating voltages from 0 to $20 \mathrm{~V}$. The cross-section curves measured with acceleration differed from the curve with no acceleration by less than $10 \%$ over the entire electron energy range above about 5 or $6 \mathrm{eV}$. Below this value the curve without acceleration fell off, probably because of the effect of

${ }^{15}$ M. E. Rudd and C. E. Kuyatt, Rev. Sci. Instr. (to be published). the residual magnetic field. With $15 \mathrm{~V}$ acceleration it is believed that the analyzer operates well down to about $3 \mathrm{eV}$.

Electrons from the analyzer were focused on the first dynode of the electron multiplier detector by an electrostatic lens. Tests showed that the counting rate for various electron energies went through a broad maximum as the voltage on the focus electrode was varied. Voltages from about 100 to $300 \mathrm{~V}$ caused very little variation in counting rates and $200 \mathrm{~V}$ was chosen for the measurements.

The electron multiplier was made by removing the photocathode from a Dumont 6292 photomultiplier. However, the method of removal was modified from that of KJ. Instead of removing the end of the envelope with a glass saw and then washing with various solvents, a file cut was made at the proper place and the glass broken by touching with a hot point. This operation was carried out in a helium atmosphere to prevent poisoning of the dynodes by the air. While still in the helium atmosphere the tube was placed in its housing and connected to the analyzer which was immediately connected to the chamber and pumped out. When prepared this way, the multiplier retained a higher gain and was more stable against changes in gain. $\mathrm{KJ}$ reported a gain of $6 \times 10^{4}$ with a voltage of $3900 \mathrm{~V}$. Using the method above, electron multipliers have been obtained which have gains of about $7 \times 10^{5}$ with a voltage of $2510 \mathrm{~V}$. A light-tight housing was necessary since the tube was found to be somewhat photosensitive even after removal of the photocathode.

The first dynode was connected to a separate power supply so that its potential could be adjusted to the value giving the maximum efficiency for electron collection and counting. A curve of counting rate versus first dynode voltage showed that electrons from 0 to $250 \mathrm{eV}$ would all be counted with virtually the same efficiency when the first dynode voltage was set at $300 \mathrm{~V}$. The efficiency for counting $500-\mathrm{eV}$ electrons was down from the maximum by about $5 \%$.

A PMC-115 oil diffusion pump with a speed of 105 liters/sec and a liquid-nitrogen cold trap were used to maintain the vacuum in the system. The analyzer and detector were pumped through the electron pipe which had a number of holes opening into the outer chamber for this purpose. Gas from the inner chamber which passed through the electron slit into the electron pipe was pumped out through these same holes. Likewise, holes were provided in the proton pipe to pump out gas which entered the proton pipe through the proton aperture. The pipe connecting the chamber to the accelerator was quite long, but the pressure in it was kept at about $5 \times 10^{-6}$ Torr by pumping not only at both ends, but also with an additional pump near the middle.

With the valve below the inner chamber closed, a ratio of about 16 could be maintained between the 
target gas pressure and the outer chamber pressure. With the target gas shut off the residual gas pressure in the inner chamber was generally below $10^{-5}$ Torr. With the valve open, the ultimate pressure of the entire system was near $10^{-7}$ Torr as read on a Consolidated Model GM-110 McLeod gauge.

The nominal purity of the helium used was $99.995 \%$ and that of the hydrogen was $99.99 \%$. The gas being used was passed through a liquid-nitrogen cold trap before being admitted to the chamber. A bubbler was also provided to prevent any buildup of impurities in the line. The gas line was thoroughly flushed and evacuated between changes of gas. Measurements of target gas pressure were made with a VG-1A ionization gauge calibrated to within $5 \%$ by the McLeod gauge. Hydrogen was generally used at a pressure of about $10^{-3}$ Torr and helium at about $2 \times 10^{-3}$ Torr.

Both steady and 60-cycle components of magnetic field were annulled by the use of three mutually perpendicular pairs of Helmholtz coils. The field could be annulled to within 1 or $2 \mathrm{mG}$ at any one place, but because of gradients, fields of the order of $5 \mathrm{mG}$ may have existed over parts of the electron trajectories. However, it was determined by measurement that the horizontal component of field had a range of about $20 \mathrm{mG}$ for which the counting rate was very nearly constant even for electron energies as low as $1.5 \mathrm{eV}$, provided the $15-\mathrm{V}$ acceleration voltage was used.

\section{MEASUREMENTS}

Absorption of electrons by the gas in the chamber and in the electron pipe was appreciable and a correction was made. Since the electrons pass through regions of different pressure it was necessary first to calculate the effective path length at the chamber pressure. This was done by using standard equations for the conductances of apertures and pipes and the measured pressure ratio between the inner and outer chamber. The resultant effective path length $x$ was $4.54 \mathrm{~cm}$. Using this value and the absorption coefficients $\alpha$ measured by Normand, ${ }^{16}$ the transmission fraction $t$ was calculated for each electron energy and each chamber pressure $p$ using the relation $t=e^{-\alpha p x}$. The values of $t$ for the various electron energies ranged from about 0.85 to 1.0 for helium and from about 0.75 to 1.0 for hydrogen at the pressures used.

The number of electrons $N_{g}$ of the proper energy and direction which originate in the target gas was calculated from the data as follows. Let $N_{2}$ be the number of counts recorded when the target gas is in the chamber and let $N_{1}$ be the number recorded when only the residual gas remains. Assume a number $N_{n}$ of noise pulses originating in the electronic circuits. Let $N_{r}$ be the number of electrons originating in the residual gas. If $t$ is the fraction of electrons transmitted by the gas, and if this fraction is essentially unity when only the re-

${ }^{16}$ C. E. Normand, Phys. Rev. 35, 1217 (1930). sidual gas is present, then we have $N_{2}=\left(N_{g}+N_{r}\right) t \eta+N_{n}$ and $N_{1}=N_{r} \eta+N_{n}$, where $\eta$ is the efficiency of the detector. This assumes that the counting rates are high enough that the statistical fluctuations are negligible. In the present investigation this was the case for all but the highest electron energies. Eliminating the unknown quantity $N_{r}$ between the two equations, one obtains

$$
\eta N_{g}=\left(N_{2} / t-N_{1}\right)-N_{n}(1-t) / t .
$$

In practice, the number of noise pulses $N_{n}$ was never appreciable compared to $N_{2}$ except at the very highest electron energies measured. But at those energies the absorption of electrons by the gas is very small and $t$ is very nearly equal to unity. In either case, the last term may be dropped and one obtains $N_{g}=\left(N_{2} / t-N_{1}\right) / \eta$.

To obtain absolute values of the cross sections it was necessary to know the efficiency $\eta$ of the electron multiplier. To determine this, an auxiliary experiment was performed in which the analyzer and electron multiplier were replaced by a Faraday cup. Using the same defining slits as with the analyzer, electrons of all energies at a given angle were collected. The electron current to the unbiased cup was read on a Keithley Model 610A electrometer. The cup was made deep to avoid losing electrons by reflection. From the known solid angle subtended at the bottom of the cup by the aperture and assuming a cosine distribution of reflected electrons, calculations show that not more than about $0.8 \%$ of the reflected (and secondary) electrons would escape. To test this calculation a small magnet was held at various positions near the bottom of the cup. No increase of current was observable for any position of the magnet showing that there was essentially complete collection of electrons by the cup.

Because of the difficulty in determining an effective absorption coefficient when electrons of all energies were present, a different technique was used in the auxiliary experiment to take account of absorption of electrons by the gas. For each combination of angle and proton energy the electron current was read as a function of target gas pressure. The ratio of current to pressure plotted against pressure on semilogarithmic graph paper yielded straight lines which were extrapolated to zero pressure. The extrapolated values of the current-to-pressure ratios were used to calculate the absolute values of cross sections differential only in angle. These were compared with the integral of the corresponding differential cross sections over all electron energies to determine the efficiency of the electron multiplier. Twelve runs were made at three different proton energies, three angles, and using two different gases. The average value of the efficiency was found to be 0.778 with a probable error of 0.070 . This value was used to calculate absolute values of all cross sections.

A number of checks were made on the apparatus. One of these was to detect any possible asymmetry of the scattering geometry. Two runs were made at the $90^{\circ}$ 


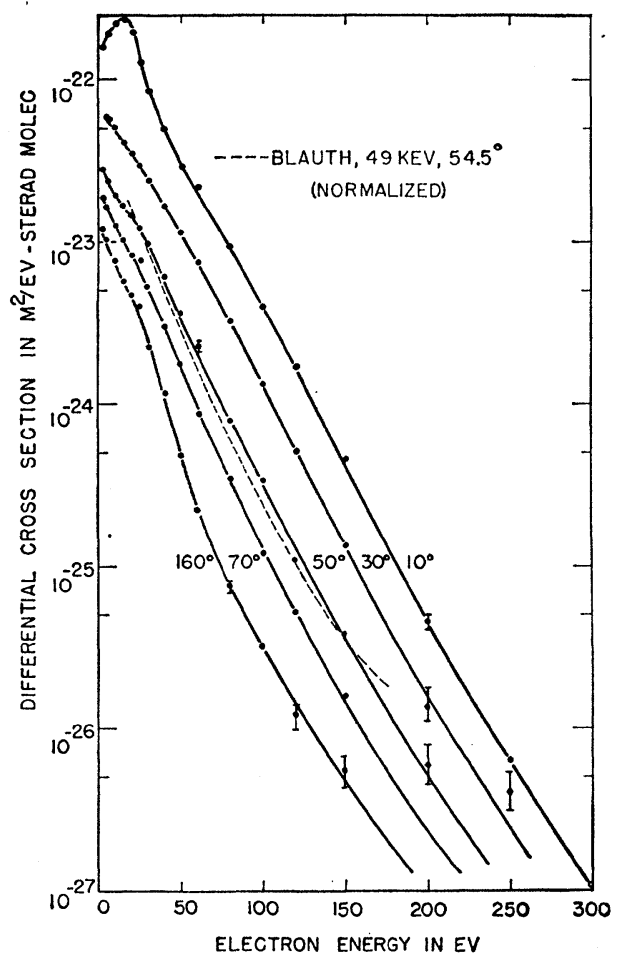

Fig. 2. Differential cross section for production of secondary electrons by $50-\mathrm{keV}$ protons in helium gas.

west port and compared to those taken at the $90^{\circ}$ east port. In both cases, the shapes of the energy distributions were practically identical at the two ports and the absolute values differed by amounts which were no greater than those experimented in successive measurements at a single port.

Tests to see if the results depended on beam current showed a slight dependence (1 to $2 \%$ for the range of currents used) at the $10^{\circ}$ port, but no dependence in measurements made at a larger angle.

The highest counting rate encountered during any run was about 11000 counts/sec. To insure that this did not overload the scaler another run was made under similar conditions but with a counting rate less than half as great. The resulting cross-section curves were very close to each other, differing at most by about $10 \%$. Furthermore, one curve was run near the beginning of the period during which the final data were taken and the other at the end. This agreement, which is within the stated uncertainty, makes it seem unlikely that any change took place during the runs which would materially affect the results.

Differential cross sections were calculated using Eq. (2) of KJ. Since the widths $w_{1}$ and $w_{2}$ of the entrance and exit slits of the analyzer were 0.3135 and $0.2429 \mathrm{~cm}$, respectively, the effective transmission $\tau$ was 0.775 and the effective resolution $\Delta E / E$ was 0.0570 .

\section{EXPERIMENTAL RESULTS}

Differential cross sections for helium are plotted in Figs. 2, 3, and 4 and for hydrogen in Fig. 5. Above about 2 or $3 \mathrm{eV}$ nearly all of the curves show a monotonic decrease in cross section as electron energy is increased. Below about $2 \mathrm{eV}$ most of the cross sections decreased due to the previously mentioned distortion produced by the analyzer at low-electron energies. Therefore, the curves are plotted down to only about $2.5 \mathrm{eV}$. The curves for some of the larger angles are omitted for clarity.

The "humps" on the $10^{\circ}$ curves were thought to be due to spurious electrons, but reruns with additional shields failed to eliminate them.

The shapes of the hydrogen curves agrees very well with the corresponding results of $\mathrm{KJ}$ above about $8 \mathrm{eV}$. Below this energy their cross sections drop off while the present results continue to increase down to about $2 \mathrm{eV}$. The reason for the discrepancy is probably the following. In their apparatus there was no cold trap between the pumps and the scattering chamber as in the present work, but each ionization gauge had a small cold trap. Thus, the gauges did not read the total pressure but only the partial pressure of the noncondensable gases. Since their chamber had rather large areas of greased rubber diaphragms, there was an appreciable amount of oil and grease vapor present. Low-energy electrons were probably absorbed strongly enough by this vapor to account for the decrease in cross section noted.

Fairly direct comparison is also possible with the work of Blauth. ${ }^{13} \mathrm{He}$ investigated the energy distribu-

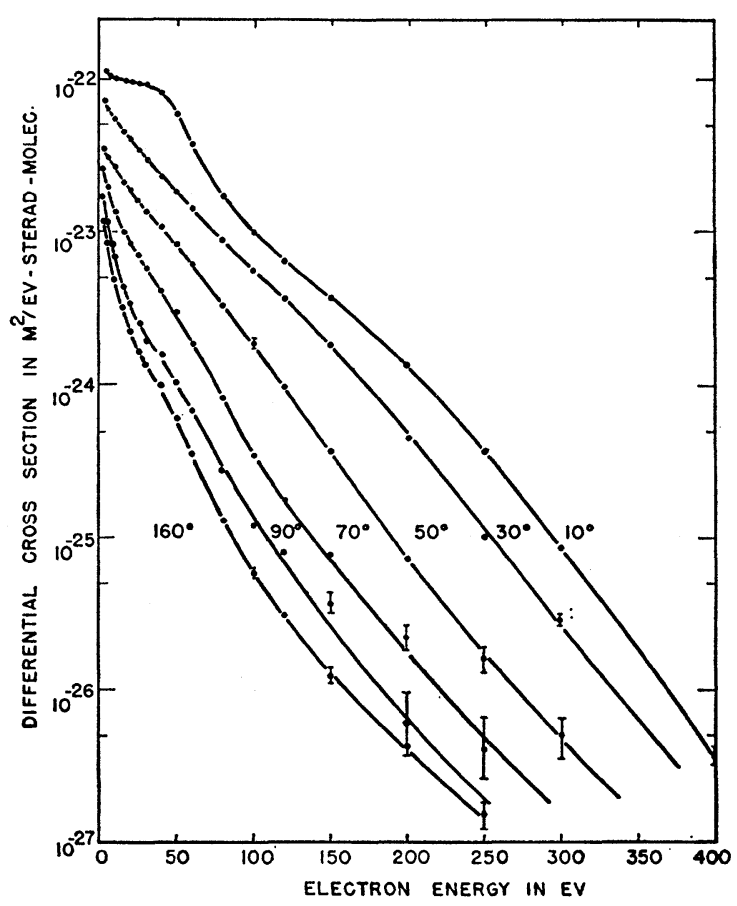

FIG. 3. Differential cross section for production of secondary electrons by $100-\mathrm{keV}$ protons in helium gas. 


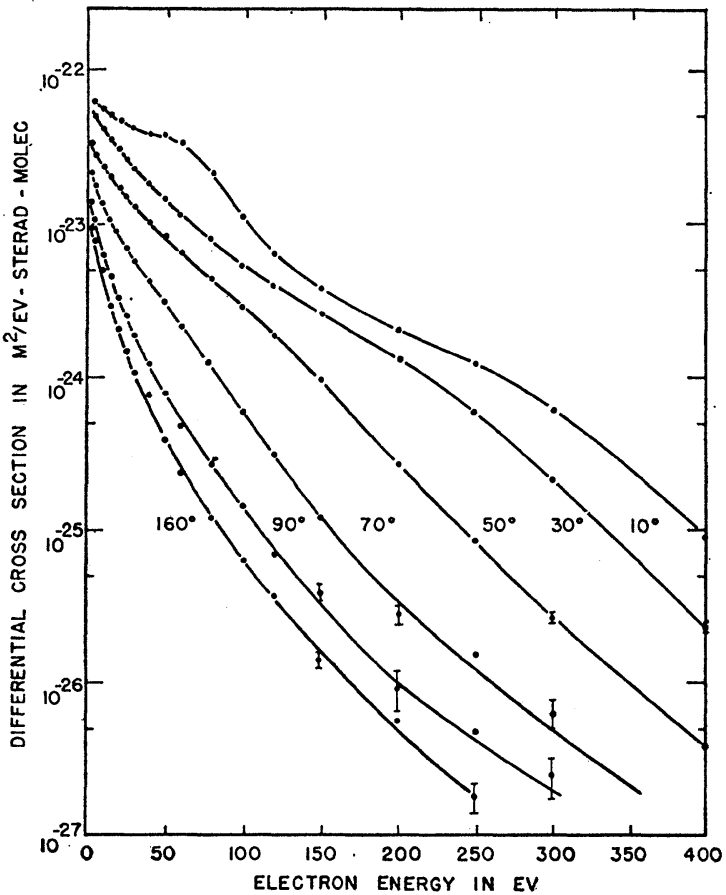

FIG. 4. Differential cross section for production of secondary electrons by $150-\mathrm{keV}$ protons in helium gas.

tion of electrons from various gases including hydrogen and helium but only at one angle of ejection, $54.5^{\circ}$. Although his helium results at $49-\mathrm{keV}$ proton energy were not published, he has very kindly supplied these data. ${ }^{17}$ Since he did not calculate cross sections but only numbers proportional to cross sections, his data have been normalized and compared with the present $50-\mathrm{keV}$ results in Fig. 2. Agreement is very good between about 25 and $130 \mathrm{eV}$.

The two largest sources of error in the relative values of the differential cross sections are the statistical counting error and the uncertainty in the electron absorption correction. The latter error is about $3 \%$ at $10 \mathrm{eV}$, somewhat greater at lower energies, and much smaller at high-electron energies. Typical values of the statistical counting error are $1 \%$ at $2 \mathrm{eV}, 2 \%$ at $50 \mathrm{eV}$, and $4 \%$ at $100 \mathrm{eV}$. The combined effect of these two sources of error on the relative cross sections is shown by error bars on some of the points on the graphs.

In addition to the errors in the relative values of the differential cross sections, the absolute values had errors associated with the calibration of the ionization gauge $(5 \%)$ and with the determination of the multiplier efficiency $(9 \%)$. When combined in $\mathrm{rms}$ fashion the total error in the absolute values is about $10 \%$.

A number of quantities of interest may be obtained from the differential cross sections by integrating the data in various ways. Equations are given by $\mathrm{KJ}$ for calculation of the cross sections differential in angle only

\footnotetext{
${ }^{17}$ E. Blauth (private communication).
}

and electron energy only, the total ionization cross section, and the average energy of the ejected electrons.

Figure 6 shows the energy distribution of electrons from helium after integrating over all angles. Figure 7 shows the same thing for hydrogen compared with the results of $\mathrm{KJ}$. The same drop in cross section below $8 \mathrm{eV}$ is noted here as before. Also, it is seen that their cross sections are about $20-25 \%$ higher than the present results above $8 \mathrm{eV}$. The reason is that since they did not obtain absolute values of cross sections, the results were normalized by comparison of the integrated cross sections with the total cross section of Schwirzke ${ }^{9}$ at $50 \mathrm{keV}$. Because of the low cross sections at low electron energies, this required higher cross sections at other energies to yield the same area under the curve.

Angular distributions of ejected electrons are shown in Figs. 8 and 9. In all cases there is a monotonic decrease in cross section with increase in angle of ejection although for helium the cross section is virtually constant above about $110^{\circ}$. It was noted that for helium over the proton-energy range studied, the lower the proton energy the more electrons are emitted at angles near $0^{\circ}$ and $180^{\circ}$ and the fewer in the intermediate range from $30^{\circ}$ to $70^{\circ}$. The same effect was noted by KJ for hydrogen.

Values obtained for the total ionization cross section are given in Table I and for helium are also plotted in Fig. 10 along with the results of other experiments. The values for helium are in excellent agreement with the results of Fedorenko et al. ${ }^{10}$ and in very good agreement with the $150-\mathrm{keV}$ point of Hooper et al. ${ }^{11,12}$ The single value of total cross section for hydrogen agrees very well

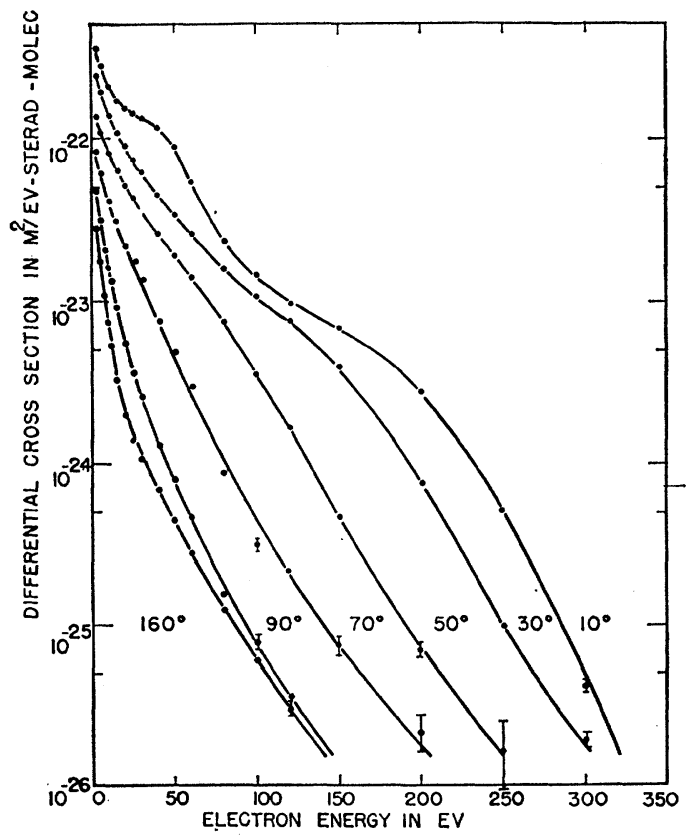

FIG. 5. Differential cross section for production of secondary electrons by $100-\mathrm{keV}$ protons in hydrogen gas. 


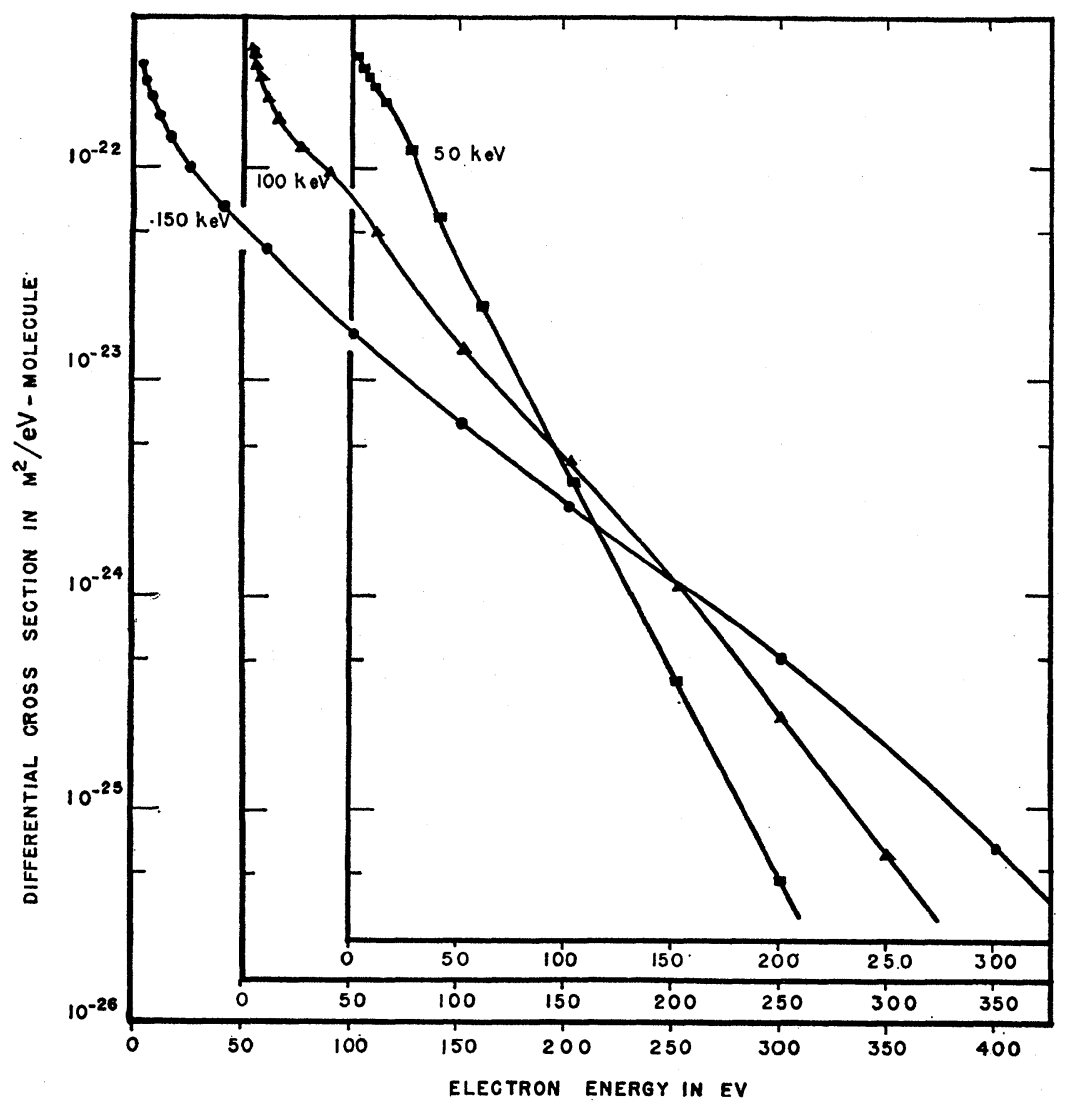

FIG. 6. Differential cross section for ejection of electrons at all angles as a function of electron energy for 50-, $100-$, and $150-\mathrm{keV}$ protons in helium gas. The 50 - and $100-\mathrm{keV}$ curves are displaced to the right to avoid overlapping.

with the results of Afrosimov et al. ${ }^{8}$ and is within the experimental error of the value given by $\mathrm{KJ}$.

In addition to the error of $10 \%$ in the absolute value of the differential cross section, cross sections which have been integrated over angle or energy or both are subject to the additional error associated with drawing the curves and finding the areas. As a check, the total cross sections were each calculated two ways; once by integrating first over electron energy and then over

TABLE I. Values of quantities calculated from the measured differential cross sections with some theoretical results for comparison.

\begin{tabular}{lcccc}
\hline \hline \multicolumn{1}{c}{ Gas } & Hydrogen & Helium & Helium & Helium \\
\hline Proton energy (keV) & 100 & 50 & 100 & 150 \\
$\sigma_{t^{\mathbf{a}}}\left(10^{-21} \mathrm{~m}^{2} /\right.$ molecule $)$ & 21.3 & 8.27 & 9.51 & 8.36 \\
$\sigma_{t}{ }^{\mathrm{b}}\left(10^{-21} \mathrm{~m}^{2} /\right.$ molecule $)$ & 16.6 & 8.8 & 8.2 & 7.1 \\
$E_{\mathrm{av}}(\mathrm{eV})$ & 28.2 & 22.6 & 35.2 & 43.0 \\
$\Delta \varepsilon_{\mathrm{av}}(\mathrm{eV})$ & 43.8 & 47.2 & 59.8 & 67.6 \\
$(1 / n)(d E / d x)$ & & & & \\
$\left(10^{-19} \mathrm{~m}^{2}-\mathrm{eV} /\right.$ molecule $)$ & 9.32 & 3.91 & 5.75 & 5.66 \\
$(1 / n)(d E / d x)^{\mathrm{f}}$ & & & & \\
$\left(10^{-19} \mathrm{~m}^{2}-\mathrm{eV} /\right.$ molecule $)$ & 9.55 & 5.46 & 6.20 & 5.73 \\
\hline \hline
\end{tabular}

a Total ionization cross section calculated from present data. d Average energy lost by a proton in an ionizing collision calculated from present data.

e Stopping cross section due to ionization calculated from present data. f Stopping cross section due to ionization calculated from the Gryzinsk theory. angle, and then by doing the integrations in the reverse order. In three cases, the average deviation of the average cross section was $1 \%$ and it was less than $\frac{1}{2} \%$ in the remaining case. In view of this excellent agreement it is believed that any error in integration was small. Furthermore, the integration involves some averaging which tends to smooth out random variations. Because of this and because of the good agreement with other experimental results, it is believed that the total cross sections may be assigned an uncertainty of $8 \%$ and the cross sections differential in angle or energy only an uncertainty of $10 \%$.

Table I contains values for $E_{\text {av }}$ the average energy of an ejected electron for the three proton energies in helium and the one in hydrogen. The number is considerably smaller than that given by $\mathrm{KJ}$ because of the aforementioned discrepancy at low electron energies.

Also listed in Table $I$ are the values of the average energy lost by a proton $\Delta \mathcal{E}_{\mathrm{av}}$ which were calculated from the relation $\Delta \mathcal{E}_{\mathrm{av}}=E_{\mathrm{av}}+U$, where $U$ is the ionization potential of a molecule. The values of $U$ were taken to be $15.6 \mathrm{eV}$ for hydrogen and $24.6 \mathrm{eV}$ for helium. The stopping cross section due to ionization is given by the relation

$$
(1 / n)(d E / d x)_{i}=\int_{0}^{\infty}(E+U) \sigma(E) d E
$$


where $\sigma(E)$ is the ionization cross section differential in energy only. These results also appear in Table I.

\section{COMPARISON WITH THEORY}

The first Born approximation has been employed by $\mathrm{KJ}$ to arrive at an expression which may be integrated numerically to obtain differential cross sections for ejection of electrons from hydrogen atoms by protons. Some results of such calculations were reported by KJ. Additional computations have now been made for a wider range of angles and electron energies to compare

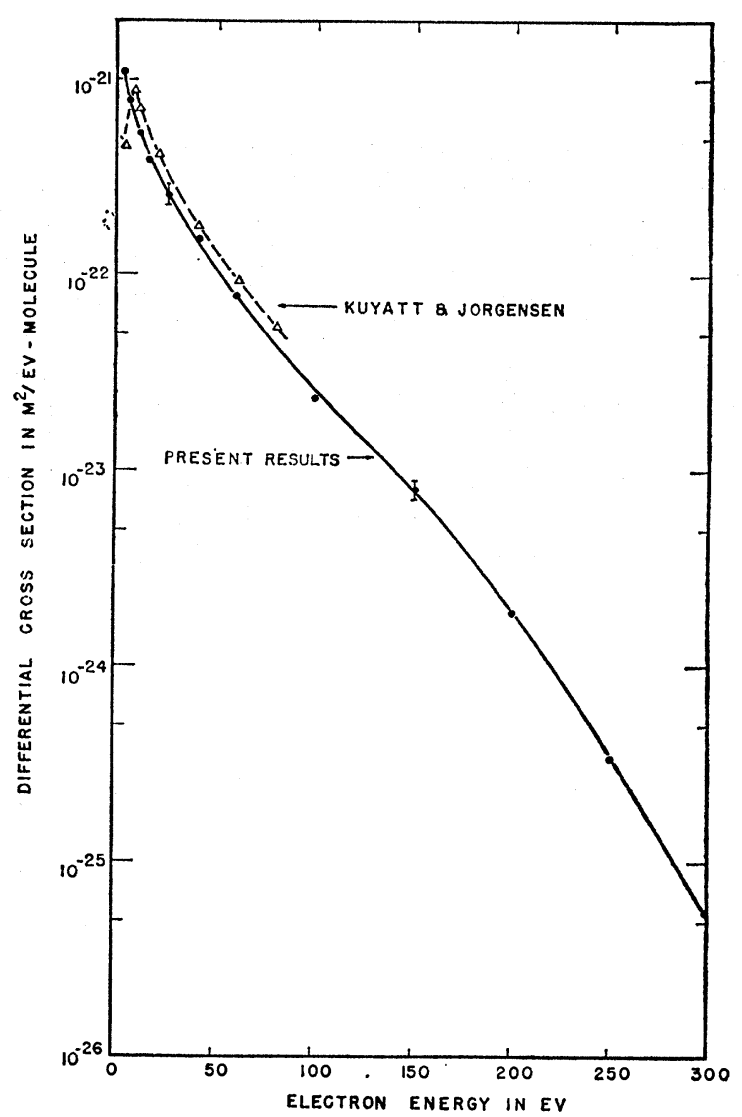

FIG. 7. Differential cross section for ejection of electrons at all angles as a function of electron energy for $100-\mathrm{keV}$ protons in hydrogen.

with the present results. The cross sections have been scaled to apply to molecular hydrogen using the procedure given by Bates and Griffing ${ }^{1}$ and used by Hooper et al..$^{11}$ The results are presented in Table II for $100-\mathrm{keV}$ protons and may be compared to the present experimental results given in Fig. 5. The general agreement is poor, but some of the features of the experimental curves are reproduced by the theory. The angular distribution is very different from experiment at low electron energies, but better at higher energies. The energy distributions predicted by the theory are gener-

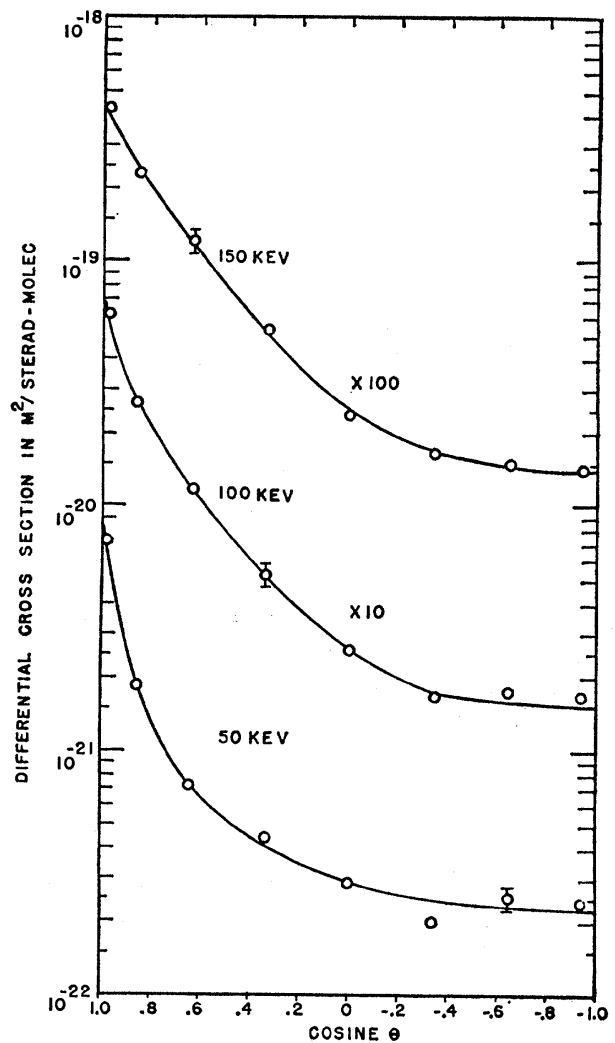

FIG. 8. Differential cross section for ejection of electrons of all energies as a function of the cosine of the angle of ejection for 50 -, $100-$, and $150-\mathrm{keV}$ protons in helium gas. The 100- and $150-\mathrm{keV}$ curves are multiplied by 10 and 100 , respectively.

ally too steep but at small angles have a region in which the curves nearly become level. The results of the theory improve considerably when the cross sections are integrated over all angles. Figure 11 shows the results of this integration in comparison with the present experimental results. Agreement is good at low electron

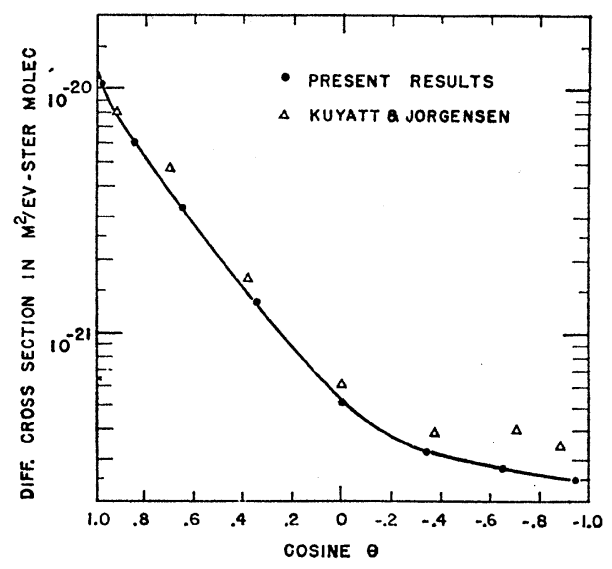

FIG. 9. Differential cross section for ejection of electrons of all energies as a function of the cosine of the angle of ejection for $100-\mathrm{keV}$ protons in hydrogen gas. 
TABLE II. Values of the differential cross section for ejection of secondary electrons from molecular hydrogen by $100-\mathrm{keV}$ protons calculated from the Born approximation. Cross sections are in units of $10^{-26} \mathrm{~m}^{2} / \mathrm{eV}$-sr-molecule.

\begin{tabular}{|c|c|c|c|c|c|c|c|}
\hline $\begin{array}{c}\text { Electron } \\
\text { energy } \\
(\mathrm{eV})\end{array}$ & $0^{\circ}$ & $10^{\circ}$ & $30^{\circ}$ & $50^{\circ}$ & $70^{\circ}$ & $90^{\circ}$ & $160^{\circ}$ \\
\hline 3.4 & 15070 & 15180 & 15740 & 15500 & 12960 & 9005 & 5425 \\
\hline 8.6 & 7560 & $\ldots$ & & $\ldots$ & & & 1487 \\
\hline 13.6 & 4528 & 4653 & 5600 & 6619 & 5218 & 2343 & 586 \\
\hline 30.5 & 1414 & 1483 & 2080 & 2842 & 1656 & 405 & 63.4 \\
\hline 54.3 & 639 & 683 & 1078 & 1322 & 374 & 58.2 & 8.05 \\
\hline 84.7 & 442 & 501 & 736 & 471 & 58.0 & 8.63 & 1.17 \\
\hline 122 & 410 & 431 & 434 & 90.3 & 7.76 & 1.403 & \\
\hline 165 & 293 & 274 & 112 & 10.8 & 1.046 & 0.232 & \\
\hline 217 & 65.8 & 54.4 & 12.1 & 1.10 & 0.139 & 0.0351 & \\
\hline 274 & 5.43 & 4.39 & 0.976 & & & & \\
\hline 338 & 0.375 & & & & & & \\
\hline
\end{tabular}

energies but becomes poorer at higher energies. The theoretical cross section is low by a factor of 2 at $150 \mathrm{eV}$ and by a factor of 5 at about 250 or $300 \mathrm{eV}$.

Bates and Griffing ${ }^{1}$ have also obtained the energy distribution of the ejected electrons from hydrogen atoms after integration over all angles. However, these results cannot be easily compared with the present experimental values since no theoretical results are given for any proton energy near $100 \mathrm{keV}$. However, two checks were made which showed that when integrated the $\mathrm{KJ}$ equation yields results identical to those of Bates and Griffing.

Using the Born approximation, Mapleton ${ }^{2}$ has calculated total ionization cross sections for helium bombarded by protons of various energies. These are plotted in Fig. 10. The numbers he obtained at an intermediate step in his calculations can be used to compute the cross sections differential in electron energy. These he generously supplied ${ }^{18}$ and the results are plotted in Fig. 12. Since the calculations were done for a proton energy of $125 \mathrm{keV}$, the 100 - and $150-\mathrm{keV}$ experimental data were averaged for the comparison. Good agreement is obtained over a wide range of electron energies.

The fact that the Born approximation appears to yield better results when applied to helium than to

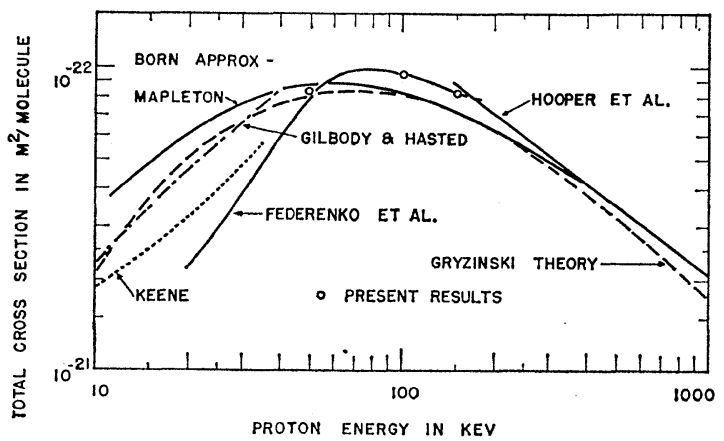

FIG. 10. Total ionization cross section for protons in helium gas as a function of proton energy. Results of other experiments and theory are shown for comparison.

${ }^{18}$ R. A. Mapleton (private communication). hydrogen lends support to the idea that the discrepancy between theory and experiment for hydrogen is not so much due to a failure of the Born approximation itself as to the dissimilarity of the hydrogen molecule and two hydrogen atoms, as suggested by $\mathrm{KJ}$ and others. It is also interesting that the Born approximation yields at least fair electron energy distributions, but poor angular distributions. Additionally, it may be noted that agreement with experiment becomes progressively better as the differential cross sections are successively integrated over angle and energy yielding fairly good values for the total ionization cross sections.

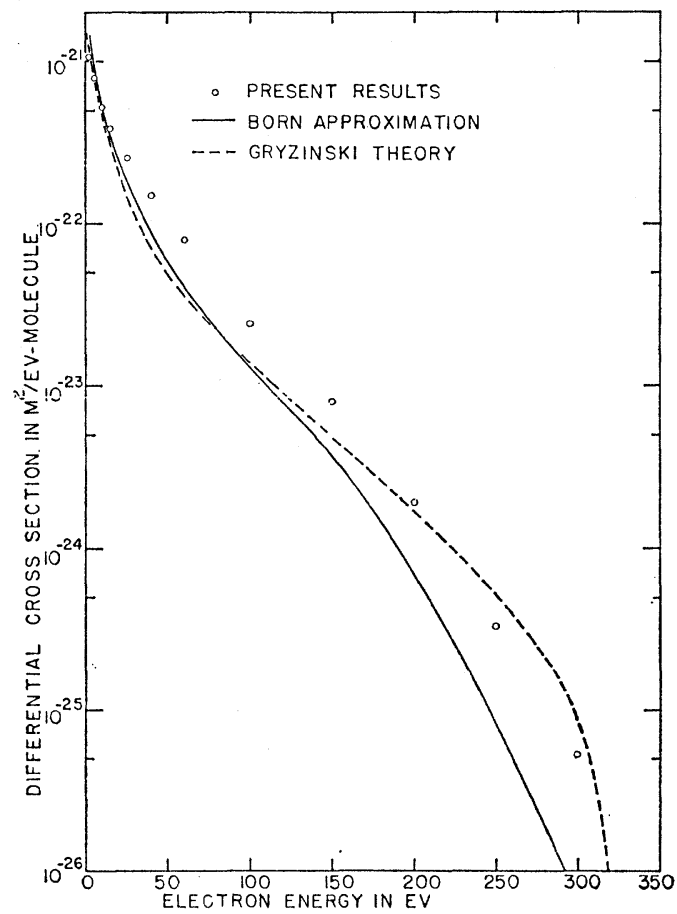

FIG. 11. Differential cross section for ejection of electrons at all angles for $100-\mathrm{keV}$ protons in hydrogen gas. Comparison is made with calculations made from the Born-approximation equation of Kuyatt and Jorgensen and with the results of the Gryzinski theory. 


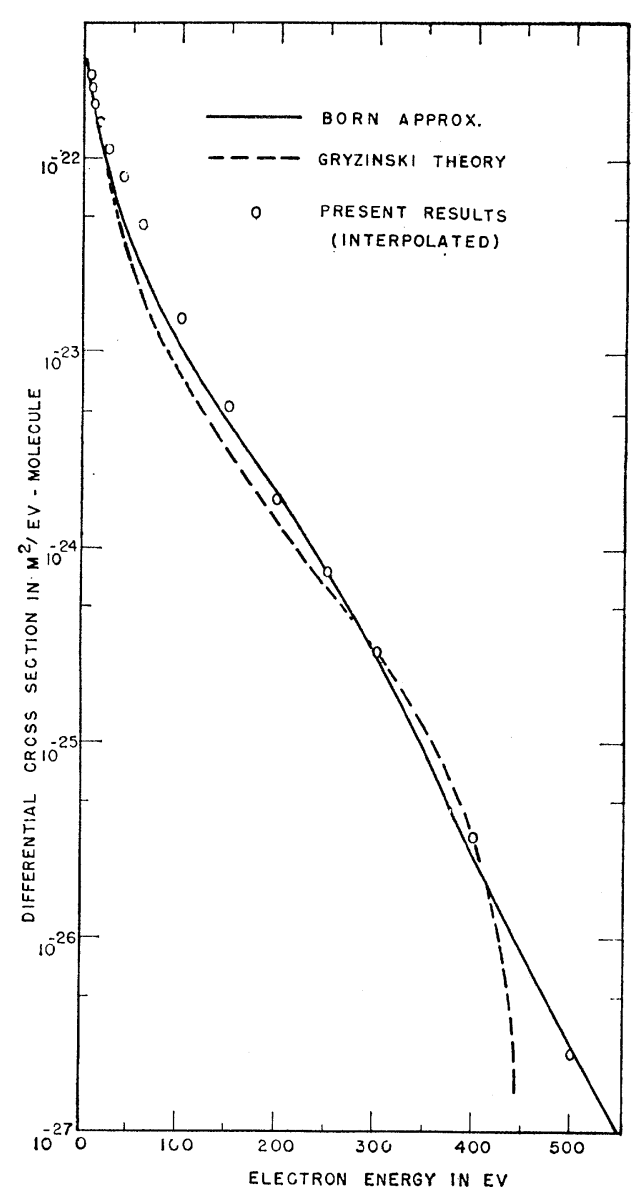

FIG. 12. Differential cross section for ejection of electrons at all angles for $125-\mathrm{keV}$ protons in helium gas. Comparison is made with Born-approximation calculations of Mapleton and with the results of the Gryzinski theory.

Gryzinski ${ }^{19}$ has proposed a classical approach to the general problem of atomic and electronic collisions. According to his treatment, one considers the proton colliding only with the electron, which is then ejected with an energy given by the value it has just after the collision minus the ionization potential of the molecule. After integrating over impact parameters and angles of

${ }^{19}$ M. Gryzinski, Phys. Rev. 115, 374 (1959). encounter he arrives at expressions for total ionization cross section and also for the energy distribution of ejected electrons. The theory is attractive since its results are in algebraic form and require no numerical integration. Calculations of cross sections differential in energy only are shown for hydrogen in Fig. 11. Agreement with experiment is only fair. Agreement is slightly better for helium where the Gryzinski theory predicts values of cross sections differential in electron energy which are within a factor of 2 of the experimental values over practically all of the electron energy range as shown in Fig. 12. However, the Born approximation results are better as shown on the same graph. As seen in Fig. 10 the Gryzinski theory yields a curve of total cross sections which is not too much different from that given by the Born approximation. However, the Gryzinski curve diverges from the experimental curve at large proton energies, whereas the Born approximation calculations of Mapleton ${ }^{2}$ are essentially identical with the experimental curve of Hooper et $a l_{0}^{11,12}$ at energies above about $400 \mathrm{keV}$. The Gryzinski theory will also supply values of stopping cross sections and these have been calculated for the proton energies treated in this investigation. The results are in Table I. Good agreement is obtained with experiment in three of the four cases.

It may be concluded that the Gryzinski theory might be useful in situations where ease of computation is more important than great accuracy, but that somewhat more reliable results are to be expected from the Born approximation.

\section{ACKNOWLEDGMENTS}

It is a pleasure to acknowledge the many valuable suggestions made by Dr. Chris E. Kuyatt. The authors wish to thank Donald McArthur for doing the computations for the Born-approximation calculation in hydrogen and Wayne Lang for doing some of the Gryzinskitheory calculations. D. J. Fuehring and Earl Brooks constructed the scattering chamber with skill and precision. Grateful appreciation is extended to Dr. Robert Mapleton for supplying the results for the Bornapproximation calculation for helium, and to Dr. Erich Blauth for his unpublished helium-ionization data. 